\title{
Record Lifetime for a Bubble
}

\author{
Researchers created a gas bubble that lived for 465 days, a world record \\ for this type of object.
}

By Katherine Wright

S oap bubbles are transient objects, lasting at most a few minutes. Now, Aymeric Roux from University of Lille, France, and colleagues have created bubbles that maintain their structures for more than 200,000 times as long [1]. The long-lived bubbles, which have a layered shell structure made from plastic particles, glycerol, and water, could be used to create stable foams.

The team studied a type of bubble known as a gas marble (see Focus: "Gas Marbles" Store Air in Strong Spheres). Such bubbles are typically made from a liquid solution that contains plastic beads. The beads pack together on the bubble's shell, making the bubble strong enough that it can be held in a hand or rolled along a surface. Researchers have explored the mechanical properties of gas marbles but not, until now, their lifetimes.

For their experiments, the team studied three different types of bubble: traditional soap bubbles, water-based gas marbles, and water-glycerol-based gas marbles. They monitored the bubbles using a balance and a camera. As expected, the soap bubbles

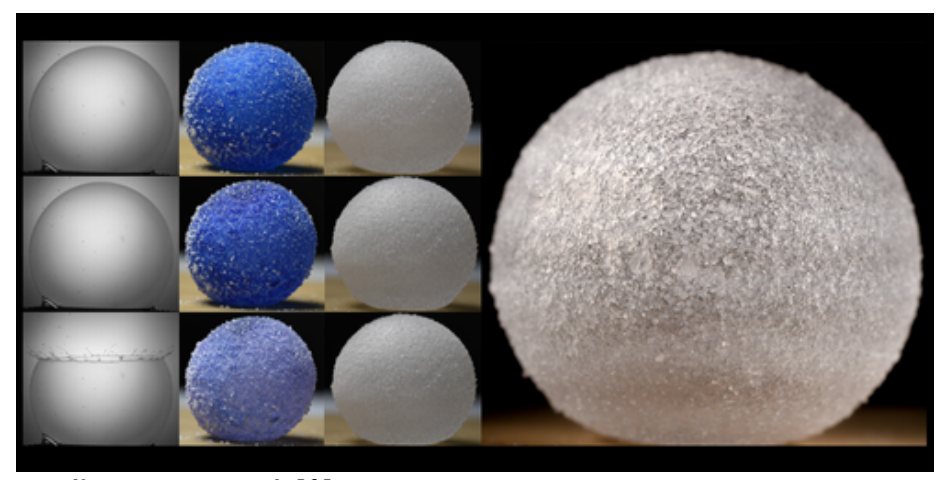

lived for around a minute before bursting, while the water-based gas marbles lasted a little longer, collapsing at the 6-to-60-minute mark. With a high enough glycerol concentration, water-glycerol marbles remained intact for significantly longer, with the longest-lasting one surviving for 465 days after it was made.

The team says that the increased longevity of the water-glycerol marbles comes from the stabilizing effects of the glycerol. Glycerol has a strong affinity with water and is known to absorb water from air. The team thinks that this absorption of water compensates for evaporation, while the presence of the particles prevents drainage of water from the shell, both of which are known causes of bubble rupture.

Katherine Wright is the Deputy Editor of Physics.

\section{REFERENCES}

1. A. Roux et al., "Everlasting bubbles and liquid films resisting drainage, evaporation, and nuclei-induced bursting," Phys. Rev. Fluids 7, L011601 (2022).

Credit: A. Roux et al. [1] 